\title{
Galois groups of prime degree polynomials with nonreal roots
}

\author{
A. Bialostocki \\ Department of Mathematics \\ University of Idaho \\ Moscow, ID, 83843. \\ and \\ T. Shaska* \\ Department of Mathematics \\ Oakland University \\ Rochester, MI, 48309-4485.
}

October 4, 2018

\begin{abstract}
In the process of computing the Galois group of a prime degree polynomial $f(x)$ over $\mathbb{Q}$ we suggest a preliminary checking for the existence of non-real roots. If $f(x)$ has non-real roots, then combining a 1871 result of Jordan and the classification of transitive groups of prime degree which follows from CFSG we get that the Galois group of $f(x)$ contains $A_{p}$ or is one of a short list. Let $f(x) \in \mathbb{Q}[x]$ be an irreducible polynomial of prime degree $p \geq 5$ and $r=2 s$ be the number of non-real roots of $f(x)$. We show that if $s$ satisfies $s(s \log s+2 \log s+3) \leq p$ then $\operatorname{Gal}(f)=A_{p}, S_{p}$.
\end{abstract}

\section{Introduction}

Solving algebraic equations is one of the oldest and most fundamental problems in mathematics. The problem was put on a firm basis with the contribution of E. Galois and was the main motivation for the development of modern algebra. It is now considered basic knowledge that a polynomial equation with rational coefficients can be solved by radicals if and only if its Galois groups is solvable. Hence, the problem of determining Galois groups is important and has

\footnotetext{
*Supported by the NSA grant R1-05-0129
} 
been considered in many areas of mathematics as number theory, group theory, algebraic geometry, and differential equations.

However, computing Galois groups is still a difficult task. Even with the development of new computer algebra systems this remains a challenge and can be accomplished only for small degree polynomials. For example, Maple 9 can only handle polynomials of degree $\leq 9$ and Kant up to degree 15. Other computer algebra packages can handle polynomials whose degree is in the same range.

Let $f(x)=a_{0}+a_{1} x+\cdots+a_{n} x^{n}=0$ be an algebraic equation. Suppose that $a_{i}= \pm 1$; then Littlewood and Offord proved that almost all these equations have less than $25\left(\log n^{2}\right)$ real roots; see 3 . If the $a_{i}$ have normal distribution with density $e^{-u^{2}} / \pi^{\frac{1}{2}}$; then Kac (1944) showed that the mean value of the number of real roots of $f(x)$ equals

$$
(4 / \pi) \int_{0}^{1} \frac{\left[1-n^{2}\left[x^{2}\left(1-x^{2}\right) /\left(1-x^{2 n}\right)\right]^{2}\right]^{\frac{1}{2}}}{1-x^{2}} d x \sim(2 / \pi) \log n,
$$

see [7. If the coefficients are random variables with distribution function $\sigma(u)$ then in a second paper Kac (1949) gives an expansion for the average number of roots of the above equation in the interval $(a, b)$. He then considers the special case when $\sigma(u)=\frac{1}{2} u$, and deduces from the general formula that in this case the average number of real roots is asymptotic to $(2 \pi)^{-1} \log n$. Consequently, polynomials in general have plenty of non-real roots.

The existence of non-real roots of a polynomial makes the computation of its Galois group much easier. Computing the Galois group in this case for polynomials of prime degree $p$ will be the focus of this short note. Checking whether a polynomial has non-real roots is very efficient since numerical methods can be used. Once the existence of non-real roots is established then from a theorem of Jordan (1871) it follows that if their number is "small" enough with respect to the degree $p$ of the polynomial, then the Galois group is $A_{p}$ or $S_{p}$. Furthermore, using the classification of finite simple groups we know nowadays the complete classification of transitive groups of prime degree. This enables us to provide a complete list of possible Galois groups for every polynomial of prime degree $p$ which has non-real roots.

In section 2 we briefly describe the existing techniques used to compute the Galois group of a polynomial $f(x) \in \mathbb{Q}[x]$ of degree $n$. These techniques are mainly based on the Dedekind's theorem and knowledge of the list of all transitive subgroups of $S_{n}$ and the cycle structure of their elements. First, a factorization $\bmod p$ (i.e., good prime $p$ ) of $f(x)$ is required to obtain information on the cycle structure of the group elements. Such information enables us to eliminate groups from the list of transitive subgroups of $S_{n}$. For as long as the group is not uniquely determined we repeat the procedure with a different prime $p$. This is a rather expensive technique since algorithms of factorizing polynomials are not very efficient. Furthermore, many primes $p$ might be needed in the process. 
In section 3 we study polynomials of prime degree $p$ with non-real roots. We describe the transitive groups of $S_{p}$ and compute all such groups for $n \leq 30$. Using a theorem of Jordan on permutation groups we show that for a fixed number of non-real roots $r=2 s$ and

$$
p \geq N(r):=[s(s \log s+2 \log s+3)]
$$

the Galois group is $A_{p}$ or $S_{p}$. Furthermore, we classify all groups that occur for $p<N(r)$ and provide an algorithm that computes the Galois group of a polynomial with non-real roots of degree prime $p$. We conclude with some final remarks in section 4 .

Notation: Throughout this paper the ground field $k=\mathbb{Q}$. All polynomials are assumed to be irreducible over $\mathbb{Q}$. The group $D_{n}$ denotes the dihedral group of $n$ elements, $M_{11}$ and $M_{23}$ are respectively Mathew groups of degree 11 and 23. For all other groups we use the GAP notation.

\section{The Galois group of a polynomial}

Let $f(x) \in k[x]$ be a degree $n$ polynomial. A splitting field of $f$ is a field extension of $k$ of the form $k\left(\alpha_{1}, \ldots, \alpha_{n}\right)$ where $f(x)=\left(x-\alpha_{1}\right) \cdots\left(x-\alpha_{n}\right)$. Any two splitting fields of $f$ are isomorphic under an isomorphism trivial on $k$. Thus we normally speak of the splitting field of $f$.

If the discriminant $\Delta_{f}$ of $f(x)$ is nonzero then $f(x)$ has $n$-distinct roots $\alpha_{1}, \ldots, \alpha_{n}$ in the splitting field $E_{f}$ of $f . E_{f} / k$ is a Galois extension. The Galois group of $f$ over $k$, denoted by $G_{k}(f)$, is the group $G\left(E_{f} / k\right)$, viewed as a permutation group of the roots $\alpha_{1}, \ldots, \alpha_{n}$. Thus $G_{k}(f)$ is a subgroup of $S_{n}$, determined up to conjugacy by $f$. The following is elementary and we avoid the proof.

Proposition 1. Let $f(x) \in k[x]$ be a degree $n$ polynomial and $G=G_{k}(f)$.

(i) Let $H=G \cap A_{n}$. Then $H=G\left(E_{f} / k\left(\sqrt{\Delta_{f}}\right)\right)$. In particular, $G$ is contained in the alternating group $A_{n}$ if and only if the discriminant $\Delta_{f}$ is a square in $k$.

(ii) The irreducible factors of $f$ in $k[x]$ correspond to the orbits of $G$. In particular, $G$ is a transitive subgroup of $S_{n}$ if and only if $f$ is irreducible.

Remark 1. Recall that for a degree $n$ irreducible polynomial $f(x)$ over $k$ with splitting field $E_{f}$ we have $n \mid\left[E_{f}: k\right]$.

Hence, for a given polynomial $f(x)$ of degree $n$ its Galois group must satisfy:

i) $G$ is isomorphic to a transitive subgroup of $S_{n}$.

ii) $n$ divides $|G|$,

iii) $G$ is a subgroup of $A_{n}$ if and only if $\Delta_{f}$ is a square in $\mathbb{Q}$.

These conditions narrow down the possible choices of groups that can be Galois groups of $f(x)$. In order to determine precisely the group $G$ we need to determine the type of cycles in $G$. 


\section{$2.1 \quad$ Reduction mod p}

The reduction method uses the fact that every polynomial with rational coefficients can be transformed into a monic polynomial with integer coefficients without changing the splitting field.

Let $f(x) \in \mathbb{Q}[x]$ be given by

$$
f(x)=x^{n}+a_{n-1} x^{n-1}+\cdots+a_{1} x+a_{0}
$$

Let $d$ be the common denominator of all coefficients $a_{0}, \cdots, a_{n-1}$. Then $g(x):=$ $d \cdot f\left(\frac{x}{d}\right)$ is a monic polynomial with integer coefficients. Clearly the splitting field of $f(x)$ is the same as the splitting field of $g(x)$. Thus, without loss of generality we can assume that $f(x)$ is a monic polynomial with integer coefficients. The following theorem gives information on the cycle shape of permutations of Gal $(f)$.

Theorem 1. (Dedekind) Let $f(x) \in \mathbb{Z}[x]$ be a monic polynomial such that $\operatorname{deg} f=n, G a l_{\mathbb{Q}}(f)=G$, and $p$ a prime such that $p \nmid \Delta_{f}$. If $f_{p}:=f(x)$ $\bmod p$ factors in $\mathbb{Z}_{p}[x]$ as a product of irreducible factors of degree

$$
n_{1}, n_{2}, n_{3}, \cdots, n_{k},
$$

then $G$ contains a permutation of type

$$
\left(n_{1}\right)\left(n_{2}\right) \cdots\left(n_{k}\right)
$$

From the Chebotarev density theorem we know that if $p \rightarrow \infty$ then the distribution of factor degrees approaches the distribution of the cycle shapes in the group. Assuming that we can compute all transitive subgroups of $S_{n}$ and their cycle shapes then the above two theorems give a basis of an algorithm to determine $\operatorname{Gal}(f)$. The transitive subgroups of $S_{n}$ can be computed in GAP for all $n \leq 30$.

Remark 2. The above technique doesn't determine the Galois group uniquely in all cases. For example there are two non-isomorphic degree 8 groups with the same cycle structure. In such cases other methods such as invariants of groups are used to determine the group uniquely.

\subsection{Transitive subgroups of $S_{n}$}

By degree of a permutation group $G$ we mean the number of points moved by $G$. The degree of a permutation $\alpha \in S_{n}$ is the number of points moved by $\langle\alpha\rangle$. The minimal degree of $G$, denoted by $m(G)$, is the smallest of degrees of elements $\alpha \neq 1$ in $G$.

In the case $n$ is a prime $n=p$ then a non-solvable transitive subgroup $G$ of $S_{p}$ is doubly transitive (Burnside theorem); see [1. pg. 431] or [12 Theorem 11.7]. Using the classification of simple groups one gets a complete list of $G$ : 
Theorem 2. Let $G$ be a doubly transitive subgroup of $S_{p}$, for a prime $p$. Then $G$ isomorphic to one of the following:

(i) $A_{p}, S_{p}$,

(ii) $p=11, G=L_{2}(11)$ or $M_{11}$,

(iii) $p=23, G=M_{23}$,

(iv) $p=\frac{\left(q^{k}-1\right)}{(q-1)}$ and $L_{k}(q) \leq G \leq \operatorname{Aut}\left(L_{k}(q)\right)$.

Proof. One can check Prop. 4.4.1 in [9] for all these statements.

Thus, the above theorem classifies all transitive non-solvable subgroups of $S_{p}$. For the solvable cases we use GAP to compute them in each case for $\leq 29$. In the Table 1, we display the number of transitive subgroups and the number of non-solvable transitive subgroups of $S_{n}$ for all $n \leq 30$.

\section{Polynomials with non-real roots}

Let $f(x) \in \mathbb{Q}[x]$ be an irreducible polynomial of degree $n>5$. Denote by $r$ the number of non-real roots of $f(x)$. Since the complex conjugation permutes the roots then $r$ is even, say $r=2 s$. By a reordering of the roots we may assume that if $f(x)$ has $r$ non-real roots then

$$
\alpha:=(1,2)(3,4) \cdots(r-1, r) \in \operatorname{Gal}(f) .
$$

Since determining the number of non-real roots can be very fast, we would like to know to what extent the number of non-real roots of $f(x)$ determines $G a l(f)$. The complex conjugation assures that $m(G) \leq r$. The existence of $\alpha$ can narrow down the list of candidates for $\operatorname{Gal}(f)$. However, it is unlikely that the group can be determined only on this information unless $p$ is "large" enough. In this case the number of non-real roots of $f(x)$ can almost determine the Galois group of $f(x)$, as we will see in the next section. Nevertheless, the test is worth running for all $p$ since it is very fast and improves the algorithm overall.

\subsection{Polynomials of prime degree}

An approach of computing Galois groups of polynomials or solving for roots is to check whether or not the polynomial is decomposable. The polynomial decomposition problem can be stated as follows: given a degree $n$ polynomial $f \in k[x]$, determine whether there exist polynomials $f_{1}, f_{2}$ of degree greater than one such that $f=f_{1} \circ f_{2}=f_{1}\left(f_{2}(x)\right)$, and in the affirmative case to compute them. From the classical Lüroth's theorem this problem is equivalent to deciding if there exists a proper intermediate field in the finite algebraic extension $k(f) \subset$ $k(x)$. From the computational point of view, there are several polynomial time algorithms for decomposing polynomials. The computation of $f_{1}(x)$ and $f_{2}(x)$ only requires $O\left(n^{2}\right)$ arithmetic operations in the ground field $k$; see for instance [2]. A polynomial $f(x) \in F[x]$ is indecomposable over the subfield $F \subset k$ if and only if $f(x)$ is indecomposable over $k$. There are fast algorithms to compute the 


\begin{tabular}{||c|c|c||}
\hline \hline Deg. & Nr. of trans. groups & Nr. of unsolvable groups \\
\hline & & \\
5 & 5 & 2 \\
6 & 16 & 4 \\
7 & 7 & 3 \\
8 & 50 & 5 \\
9 & 34 & 4 \\
10 & 45 & 21 \\
11 & 8 & 4 \\
12 & 301 & 36 \\
13 & 9 & 3 \\
14 & 63 & 27 \\
15 & 104 & 40 \\
16 & 1954 & 49 \\
17 & 10 & 5 \\
18 & 983 & 91 \\
19 & 8 & 2 \\
20 & 1117 & 358 \\
21 & 164 & 56 \\
22 & 59 & 27 \\
23 & 7 & 3 \\
24 & 25000 & 807 \\
25 & 211 & 79 \\
26 & 96 & 26 \\
27 & 2392 & 64 \\
28 & 1854 & 617 \\
29 & 8 & 2 \\
30 & 5712 & 1896 \\
\hline \hline
\end{tabular}

Table 1: The number or transitive and unsolvable groups (up to conjugacy) for degree $\leq 30$

decomposition of polynomials; see 2]. However, for indecomposable polynomials we would like to have better methods of determining the Galois group and possible roots of the polynomial. Hence, polynomials of prime degree are of interest since they are, of course, indecomposable.

The next theorem determines the Galois group of a prime degree polynomial $f(x)$ with $r$ non-real roots when the degree of $f(x)$ is large enough with respect to $r$.

Theorem 3. Let $f(x) \in \mathbb{Q}[x]$ be an irreducible polynomial of prime degree $p \geq 5$ 
and $r=2 s$ be the number of non-real roots of $f(x)$. If s satisfies

$$
s(s \log s+2 \log s+3) \leq p
$$

then $\operatorname{Gal}(f)=A_{p}, S_{p}$.

Proof. Since $p$ is prime then every transitive subgroup of $S_{p}$ is primitive. Let $G$ denote the Galois group of $f(x)$ and $m(G)$ its minimal degree. By reordering the roots we can assume that

$$
(1,2)(3,4) \cdots(r-1, r) \in \operatorname{Gal}(f)
$$

Hence, $m:=m(G) \leq r$. From a theorem of Jordan [5] we have that if

$$
\frac{m^{2}}{4} \log \frac{m}{2}+m\left(\log \frac{m}{2}+\frac{3}{2}\right) \leq p
$$

then $G=A_{p}$ or $S_{p}$. Hence, if

$$
s(s \log s+2 \log s+3) \leq p
$$

then $\operatorname{Gal}(f)=A_{p}$ or $S_{p}$.

Remark 3. For a modern view of Jordan's theorem and its implications to number theory, Galois representations, and topology see [10].

For a fixed $p$ the above bound is not sharp as we will see below. However, the above theorem can be used successfully if $s$ is fixed. We denote the above bound on $p$ by

$$
N(r):=[s(s \log s+2 \log s+3)]
$$

for $r=2 s$. Hence, for a fixed number of non-real roots, for $p \geq N(r)$ the Galois group is always $A_{p}$ or $S_{p}$.

Corollary 1. Let a polynomial of prime degree $p$ have $r$ non-real roots. If one of the following holds:

(i) $r=4$ and $p>7$,

(ii) $r=6$ and $p>13$,

(iii) $r=8$ and $p>23$,

(iv) $r=10$ and $p>37$,

then $\operatorname{Gal}(f)=A_{p}$ or $S_{p}$.

Remark 4. The above results gives a very quick way of determining the Galois group for polynomials with non-real roots. Whether or not the discriminant is a complete square can be used to distinguish between $A_{p}$ and $S_{p}$.

If $p<N(r)$ then some exceptional cases occur. Next theorem determines these exceptional cases for polynomials of degree up to 29. The computations were made using GAP. 
Table 2: Galois groups (other then $A_{p}, S_{p}$ ) of polynomials with non-real roots.

\begin{tabular}{|c|c|c|c|c|}
\hline$p$ & Solv. & Sign. & Nonsol. & Sign. \\
\hline 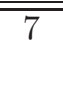 & $\begin{array}{c}D_{7} \\
(7,4)\end{array}$ & $\begin{array}{c}(2)^{3},(7) \\
(2)^{3},(3)^{2},(7)\end{array}$ & $\overline{L L(7)}$ & 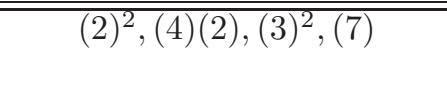 \\
\hline 11 & $\begin{array}{c}D_{11} \\
(11,4)\end{array}$ & $\begin{array}{c}(2)^{5},(11) \\
(2)^{5},(5)^{2},(10),(11)\end{array}$ & $\begin{array}{l}L(11) \\
M_{11}\end{array}$ & $\begin{array}{c}(2)^{4},(3)^{3},(5)^{2},(2)(6)(3),(11) \\
(2)^{4},(2)(6)(3),(2)(8),(3)^{3}, \\
(4)^{2},(5)^{2},(11)\end{array}$ \\
\hline$\overline{13}$ & $\begin{array}{c}D_{13} \\
(13,4) \\
(13,5) \\
(13,6)\end{array}$ & $\begin{array}{c}(2)^{6},(13) \\
(2)^{6},(4)^{3},(13) \\
(2)^{6},(3)^{4},(6)^{2},(13) \\
(2)^{6},(3)^{4},(4)^{3}, \\
(6)^{2},(12),(13) \\
\end{array}$ & $\overline{L L(13)}$ & $\begin{array}{c}(2)^{4},(3)^{3},(3)^{4},(4)^{2}(2)^{2}, \\
(6)(3)(2),(8)(4),(13)\end{array}$ \\
\hline 17 & $\begin{array}{l}D_{17} \\
(17,3)\end{array}$ & $\begin{array}{c}(2)^{8},(17) \\
(2)^{8},(4)^{4},(17)\end{array}$ & $\begin{array}{c}P S L_{2}(16) \\
(17,7)\end{array}$ & $\begin{array}{c}(2)^{8},(3)^{5},(5)^{3},(15),(17) \\
(2)^{6},(2)^{8},(3)^{5},(4)^{4} \\
(5)^{3},(6)^{2}(3),(5)(10)(2) \\
(15),(17)\end{array}$ \\
\hline & $\begin{array}{l}(17,4) \\
(17,5) \\
\end{array}$ & $\begin{array}{c}(2)^{8},(4)^{4},(8)^{2},(17) \\
(2)^{8},(4)^{4},(8)^{2},(16),(17)\end{array}$ & $(17,8)$ & $\begin{array}{c}(2)(5)(10),(2)(4),(2)(4)^{3} \\
(2)^{6},(2)^{8},(3)(6)^{2},(3)^{5} \\
(3)^{2}(12),(4)^{3},(5)^{3},(8)^{2} \\
(15),(17)\end{array}$ \\
\hline 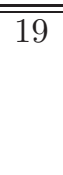 & $\begin{array}{c}D_{19} \\
(19,4) \\
(19,6)\end{array}$ & $\begin{array}{c}(2)^{9},(19) \\
(2)^{9},(3)^{6},(6)^{3},(19) \\
(2)^{9},(3)^{6},(6)^{3},(9)^{2} \\
(18),(19)\end{array}$ & & \\
\hline$\overline{23}$ & $\begin{array}{c}D_{23} \\
(23,4)\end{array}$ & $\begin{array}{c}(2)^{11},(23) \\
(2)^{11},(11)^{2},(22),(23)\end{array}$ & $\bar{M}_{23}$ & $\begin{array}{c}(2)^{8},(2)^{2}(4)^{4},(2)(7)(14), \\
(2)(4)(8)^{2},(2)^{2}(3)^{2}(6)^{2}, \\
(3)(5)(15),(5)^{3},(5)^{4}, \\
(7)^{3},(11)^{2},(23)\end{array}$ \\
\hline$\overline{\overline{29}}$ & $\begin{array}{c}D_{29} \\
(29,3) \\
(29,5) \\
(29.6)\end{array}$ & $\begin{array}{c}(2)^{14},(29) \\
(2)^{14},(4)^{7},(29) \\
(2)^{14},(7)^{4},(14)^{2},(29) \\
(2)^{14},(4)^{7},(7)^{4},(14)^{2}, \\
(28),(29)\end{array}$ & & \\
\hline
\end{tabular}

Theorem 4. Let $f(x) \in \mathbb{Q}[x]$ be an irreducible polynomial of prime degree $p>5$. Let $r$ be the number of complex roots of $f(x)$. If $r>0$ then $G a l(f)$ is $A_{p}, S_{p}$ or one of the groups as in the following Table 2. 
Proof. The proof is computational and follows from the tables of transitive subgroups of $S_{p}$. It is easy to decide which ones of these groups are nonsolvable and compute their cycle types.

Remark 5. We used in Table 2 notations which we considered standard as $D_{n}$, $M_{11}$, and $L(p)$, otherwise we used the GAP notation $(p, i)$ which is the $i$-th group in the list of transitive groups of degree $p$. These groups can be generated in GAP simple by typing TransitiveGroup(n,i);. The group $M_{23}$ is not realized as a Galois groups over $\mathbb{Q}$.

Notice that no two groups of Table 2 have the same cycle structure. Hence the Galois group can be determined uniquely by reduction mod $p$ for all polynomials of prime degree $\leq 29$ with non-real roots.

Example 1. Let $f(x)$ be the polynomial

$$
f(x)=x^{11}+5 x^{7}-4 x^{6}-20 x^{5}+4 x^{4}+20 x^{3}+1
$$

This polynomial is irreducible over $\mathbb{Q}$ and has exactly 10 non-real roots. The reader can easily check these facts in Maple using the commands:

factor $(\mathrm{f}(\mathrm{x}))$; $\operatorname{realroot}(\mathrm{f}(\mathrm{x}))$;

From the above theorem, its Galois group is $A_{11}$ or $S_{11}$. Its discriminant is

$$
\Delta_{f}=-59 \cdot 1391212936091429123033
$$

which is not a square in $\mathbb{Q}$. Hence the Galois group of $f(x)$ is $S_{11}$. Maple can not compute the Galois group of this polynomial since its degree is $>8$.

Combining the above results we have the following algorithm for computing the Galois group of prime degree polynomials with non-real roots. D $(f)$ denotes the discriminant of $f(x)$ and A_p, S_p the alternating and symmetric group of $p$ letters. Note that in the case $p<N(r)$ the we know that a permutation of the type $(2)^{\frac{r}{2}}$ is in the group. Hence, the list of transitive subgroups is much shorter than in general. This information was obtained by computing the number of real roots other then by some factorization modulo $p$. Thus, even in this case the algorithm is improved.

Algorithm: Computing the Galois group of prime degree polynomials with non-real roots.

Input: An irreducible monic polynomial $f(x) \in \mathbb{Q}[x]$ of prime degree $p$. Output: Galois group $G a l(f)$ of $f(x)$ over $\mathbb{Q}$

begin

$r:=$ NumberOfRealRoots $(\mathrm{f}(\mathrm{x}))$;

If $p>N(r)$ then 


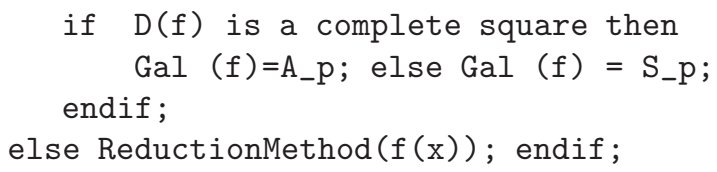

\subsection{Polynomials of prime degree $p$ with Galois group $A_{p}$}

Let $f(x)$ be a polynomial in $\mathbb{Q}(t)$ as below

$$
f(x)=(n-1) x^{n}-n x^{n-1}+t .
$$

The discriminant of $f(x)$ with respect to $x$ is

$$
\Delta_{f}=(-1)^{\frac{n(n-1)}{2}} n^{n}(n-1)^{n-1} t^{n-2}(t-1) .
$$

$\Delta_{f}$ is a complete square in $\mathbb{Q}$ if $(-1)^{\frac{(n-1)}{2}} n t(t-1)$ is a complete square; see [1] (pg. 44) for more on this family of polynomials. Let $n=23$. Then

$$
\Delta_{f}=-2^{22} \cdot 11^{22} \cdot 23^{23} \cdot t^{21}(t-1) .
$$

Hence, $\Delta_{f}$ is a complete square in $\mathbb{Q}$ if $G(t)=-23 t(t-1)$ is a complete square. In other words, for all those rational points on the curve

$$
y^{2}=G(t)
$$

This is a genus 0 curve and can be parametrized as follows:

$$
(y, t)=\left(-\frac{23 m}{m^{2}+23}, \frac{23}{\left(m^{2}+23\right.}\right)
$$

Consider $f(x)$ for $t=\frac{23}{\left(m^{2}+23\right.}$. Since we prefer to work with polynomials with integer coefficients then take

$$
f(x)=\left(22 m^{2}+506\right) x^{23}-\left(23 m^{2}+529\right) x^{22}+23 .
$$

It is easily checked that $f(x)$ is irreducible over $\mathbb{Q}$ and its discriminant is

$$
\Delta_{f}=2^{22} \cdot 11^{22} \cdot 23^{44} \cdot m^{2}\left(23+m^{2}\right)^{22}
$$

which is a complete square in $\mathbb{Q}$. Thus, $\operatorname{Gal}(f)$ is inside $A_{23}$. It is an simple calculus exercise to show that the number of real roots of these polynomials is $\leq 3$. Hence, the Galois group is $A_{23}$.

We conclude with the following open problem:

Problem: Find a degree 23 polynomial $f(x) \in \mathbb{Q}[x]$ with exactly 7 real roots such that $\Delta_{f}$ is a complete square in $\mathbb{Q}$ but $G a l(f)$ is not isomorphic to $A_{23}$.

The reader is probably aware that the above is an open problem of the inverse Galois problem, see [13. Its solution would realize $M_{23}$ as a Galois group over Q. 


\section{Concluding remarks}

The algorithm suggested in this paper works very well with prime degree polynomials which have nonreal roots. Since most polynomials have such roots this is an effective test to be implemented on all algorithms computing Galois groups. Most computer algebra packages have already algorithms implemented to find the number of real roots of a polynomial. For example in Maple the user can easily check using the command realroot $(f(x))$;. One can also generalize this algorithm to any degree $n$ polynomial. However, in this case a more detailed analysis is required.

\section{References}

[1] W. Burnside, Theory of groups of finite order. 2d ed. Dover Publications, Inc., New York, 1955.

[2] J. Gutierrez, A polynomial decomposition algorithm over factorial domains, Comptes Rendues Mathematiques, de Ac. de Sciences, 13 (1991), 81-86.

[3] J. E. Littlewood, A. C. Offord, On the number of real roots of a random algebraic equation. III. Rec. Math. [Mat. Sbornik] N.S. 12(54), (1943). $277-$ 286.

[4] Janusz, Gerald J. Permutation groups generated by a transposition and another element. Enseign. Math. (2) 38 (1992), no. 1-2, 41-53.

[5] C. Jordan, Recherches sur les substitutions, J. Liouville 17 (1872), 351-367.

[6] Kochetkov, Yu. Yu. Permutation groups generated by a $(2,2)$-cycle and an n-cycle. (Russian) Funktsional. Anal. i Prilozhen. 31 (1997), no. 2, 76-79; translation in Funct. Anal. Appl. 31 (1997), no. 2, 133-135

[7] M. Kac, On the average number of real roots of a random algebraic equation. Bull. Amer. Math. Soc. 49, (1943). 314-320.

[8] M. Kac, On the average number of real roots of a random algebraic equation. II. Proc. London Math. Soc. (2) 50, (1949). 390-408.

[9] A. Kostrikin, P. Tiep, Orthogonal decompositions and integral lattices. de Gruyter Expositions in Mathematics, 15. Walter de Gruyter \& Co., Berlin, 1994. $\mathrm{x}+535$ pp. ISBN: 3-11-013783-6.

[10] J. P. Serre, On a theorem of Jordan. Bull. Amer. Math. Soc. (N.S.) 40 (2003), no. 4, 429-440

[11] J. P. Serre, Topics in Galois theory. Research Notes in Mathematics, 1. Jones and Bartlett Publishers, Boston, MA, 1992. xvi+117 pp. ISBN 086720-210-6. 
[12] H. Wielandt, Finite permutation groups. Academic Press, New YorkLondon, 1964.

[13] H. Völklein, Groups as Galois groups. An introduction. Cambridge Studies in Advanced Mathematics, 53. Cambridge University Press, Cambridge, 1996. 